\title{
De l'envahissement à la maîtrise
}

\author{
Francine Prévost
}

\section{From Invasion to Mastery}

The first voice women hear within them is a stranger's voice. They feel invaded and this paralyzes their thought and action. The subject develops and changes when it takes risks and breaks with habits. Then, it moves to mastery of its intimate territory, freeing itself from demagogy and ideology. Demagogy is exercised in the limits the fathers place on the formulation of questions. Ideology is at work in the mothers' failure to answer. As subject, I am situated between demagogy and ideology, breaking with the philosophical tradition of the neutral knowing subject to ask and answer questions from a feminist perspective. The universality of the subject is as much a reality for me as my femaleness. This double inheritance is at work in the intimacy of the metaphors, the place where I unveil myself as subject. Here I am in the presence of Being and transcendence taking risks I have long been forbidden. Transcendence is not a reality that may be contemplated but something that is created in a going beyond. This is a silent transcendence that doesn't impose itself but rather no longer reflected, proceeds from creation to inhabit its own space.

La première voix que la femme entend au fond d'elle-même est une voix étrangère. Mais sa disponibilité à l'autre l'égare et elle croit entendre sa propre voix.

Les racines de sa pensée ne sont pas les siennes.

Elle se retrouve dans une maturité qui porte les traces des multiples autres avec qui elle a partagé le pouvoir sur des contrées intérieures qui auraient dû demeurer sous sa seule juridiction.

Elle a cru pendant longtemps que tout pouvait la pénétrer sans la détruire. Mais elle avait à cette époque la témérité des enfants malaimés. Elle croyait qu'il suffisait de combler le vide avec n'importe quoi pour arriver à $l^{\prime}$ oublier.

Elle se retrouve paralysée dans sa connaissance et dans son action. 
Au centre de ma réflexion sur les femmes et la philosophie, il y a ce sujet envahie par l'autre, cette femme disponible à toutes les questions et les réponses formulées par d'autres qu'elle-même. Cherchant à préciser ce qui, en elle, parle à sa place, ce qui l'empêche et l'entrave dans sa démarche pour sa pensée.

Fille de mères et de pères qui ont tracé les balises de son savoir et de ses expériences, elle craint de les trahir et de transgresser leurs lois si elle prend la parole en son nom propre.

Pourtant le sujet s'engendre et se métamorphose lorsqu'il parvient à quitter le giron des certitudes apprises et risque ses premiers balbutiements, lorsqu'il démasque la voix de ceux et celles qui pensent à sa place et qu'il pose enfin ses propres questions.

C'est alors seulement qu'il passe de l'envahissement à la maîtrise de son territoire c'est-à-dire qu'il devient maître de son lieu intime. Car c'est dans la formulation des questions que s'exerce le plus la démagogie de l'autre. L'idéologie est dans les réponses mais la démagogie se montre quand la parole autoritaire ne permet pas de poser certaines questions.

Au centre de mes préoccupations philosophiques, il y a moi, sujet pensant, qui se situe entre la démagogie des pères, et l'idéologie des mères.

Je me retrouve devant une tradition philosophique qui remonte à plusieurs siècles en arrière, où des hommes ont posé la question du sujet sans jamais prendre la parole au 'je', camouflant ainsi l'ancrage de leur réflexion. Une tradition qui, du même coup, a développé de multiples théories du sujet, mais rapetissé, à travers les siècles, les intérêts du sujet pensant. Surtout depuis la critique kantienne où le champ philosophique a fixé ses balises, précisé les conditions de possibilité de sa connaissance et relégué à l'arrière-scène le questionnement métaphysique.

Pour moi, qui s'intéresse à la présence de l'Être, ses énigmes et ses écueils, c'était là un piège et je me suis vue dans l'obligation de quitter au plus vite les champs épistémologiques de la philosophie critique et scientifique telle qu' elle m'avait été enseignée si je voulais survivre. Il me fallut aussi m'exercer à poser des questions qui ne camouflaient plus mes sources profondes.

Mais ces questions trouvaient déjà des réponses dans les théories du sujet au féminin et la tradition féministe. Il y avait là un autre piège pour moi. En prenant la parole au 'je', les théoriciennes du féminisme ont fait valoir la spécificité des femmes et démasqué les prétentions à 
l'universalité du discours philosophique mâle, mais elles ont du même coup forcément négligé les réponses concernant l'articulation du féminin et du masculin dans l'être humain car, celui-ci étant un universel, il introduisait du même coup une hiérarchie d'ordre patriarcal bien mal venue.

Pour moi qui s'intéresse à la présence du féminin et du masculin dans le sujet et à sa relation à l'Être, à cette jonction entre ce qui se dévoile et ce qui se cache, il me fallut là aussi quitter ce territoire et chercher en moi d'autres réponses.

Maintenant qu'il m'est donné de voir ce que je n'osais pas poser côte à côte au centre de ma parole, il me faut considérer comment tout cela se dévoile dans ma propre expérience, car elle seule peut servir de fondement à mon interrogation et à ma vision des choses.

L'universalité de l'être humain est pour moi une réalité au même titre que ma féminité. Il m'apparaît qu'au-delà de ce qui se révèle et se montre dans l'extériorité de mon sexe, il y a une intériorité qui a quelque chose à voir avec ma double appartenance. Cette intériorité demeure voilée tant que l'autre sexe ne se place pas en face de moi, à la fois, dans son extériorité et dans la double appartenance de son intériorité. Mais dès que cela se fait, mon intériorité s'élargit et commence à trouver des assises plus solides.

Cette double appartenance de l'être humain ne tient pas tant à l'androgénéité dont parlent les généticiens qu'à la présence en soi de la mère et du père qui se sont manifestés comme femme et homme et dont la double parole a creusé en nous le lieu intime de nos métaphores, ce lieu où je peux me dévoiler comme sujet.

$C^{\prime}$ est dans ce lieu de mes métaphores intimes et inédites que se révèle alors la présence de l'Être et sa transcendance. Car c'est au-delà $\mathrm{du}$ féminin et du masculin que je me cherche et $\mathrm{m}^{\prime}$ entends comme sujet extérieur et intérieur.

La question de la transcendance a pour moi quelque chose à voir avec l'expérience universelle du sujet comme lieu de gestation et de création, plutôt que lieu de réflexion. La transcendance n'est pas une réalité posée devant soi sur laquelle l'esprit peut s'attarder et réfléchir, mais elle est ce qui se fait, se fabrique, s'invente à partir du sujet, dans ce désir de dépassement du seul genre en nous qui s'expose aux regards des autres.

C'est parce que le sujet s'expérimente intérieurement dans sa double appartenance à partir de la présence en lui de sa mère et de son 
père qu'il expérimente du même coup la transcendance muette, celle qui ne le précède pas, mais celle qui procède de lui.

$C^{\prime}$ est parce que le sujet ne se voit plus comme lieu de réflexion i.e. réfléchissant la parole de la mère et du père, mais comme lieu de gestation et de création, qu'il rejoint en lui un féminin qui n'est en aucune façon une parole matriarcale aliénante et un masculin qui n'est en aucune façon une parole patriarcale aliénante.

Le sujet expérimente alors la présence d'une transcendance que je dis muette, parce que le sujet qui entend sa propre voix et ne se laisse plus envahir par l'autre, mère et père, crée des transcendances qui n'émiettent pas son identité. Contrairement aux transcendances bavardes qui parlent à sa place du sujet, s'imposent à lui dans sa réflexion et l'empêchent de créer sa propre parole parce qu'elles le précedent toujours, les transcendances muettes sont celles qui procèdent du sujet qui se constitue alors comme leur lieu de gestation et de création. C'est en puisant en elles, sources de son identité, que le sujet articule sa vision du monde, sa place dans l'Univers et le sens qu'il donne à sa vie. Toute autre transcendance, formulée par d'autres que le sujet lui-même est dangereuse pour lui, surtout quand ce sont des transcendances qui surgissent des discours démagogiques ou idéologiques de ses pères et mères et parlent à leur place.

Le sujet a besoin de s'expérimenter comme être total qui porte en lui une double appartenance pour sortir de l'envahissement du père et de la mère et passer à la maîtrise de son territoire. C'est à cette condition qu'il devient en quelque sorte sa propre autorité. Là est la véritable compétence du sujet qui prend la parole: dire ce quile transcende sur un territoire qu'il habite.

La présence de l'Etre est cette transcendance féconde mais muette que le sujet se crée en prenant la place qui lui est propre et en projetant sa parole sur la place publique.

Passer de l'envahissement à la maîtrise, c'est ne plus craindre d'aller puiser dans les profondeurs de $l^{\prime} \hat{E}$ tre, au-delà de la voix des autres, ce qui rejoint les métaphores intimes et inédites du sujet vivant. Il faut pour cela poser des questions en portant le regard vers le mystère qu'elles font émerger, plutôt que d'offrir des formulations qui cherchent à résoudre l'énigme et le figent. Il faut s'abandonner à des incertitudes et pénétrer des territoires dont on nous a pendant trop longtemps interdit l'accès. 